\title{
Preface
}

\section{PET/MR Imaging: Advances in Instrumentation and Quantitative Procedures}

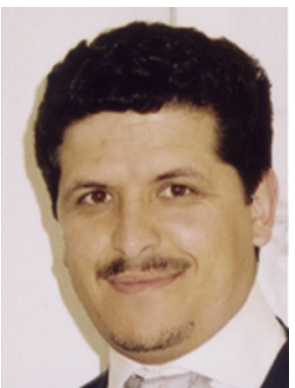

Habib Zaidi, PhD

Editor
This is an exciting time for hybrid imaging, where the success of the combination of molecular information provided by PET and structural information provided by radiographic computed tomography (CT) motivated the combination of PET with other imaging modalities, such as MR imaging, ultrasound, optical imaging, and a few other diagnostic imaging techniques. There are some interesting lessons to be learned from the history of multimodality imaging. A number of pioneers in radiological sciences realized very early (during the 1950s to 1960s) the potential and both technical and clinical benefits of combined emission and transmission imaging. ${ }^{1-3}$ In most of the situations, the clinical demand triggered technical developments of software- and hardware-based integration of various imaging modalities to respond to this need to address clinical questions. This fact explains the widespread adoption of image coregistration software, particularly in brain imaging, and integrated whole-body PET-CT scanners, which replaced stand-alone PET scanners, in clinical oncology. The rapid pace of hybrid imaging technology has been inspired by the desire of radiologists and nuclear medicine physicians to improve over existing state-of-the-art single-modality techniques in terms of clinical diagnosis, staging and restaging, therapy response monitoring, and radiation therapy treatment planning. ${ }^{4}$

The history of PET/MR imaging was an exception in the sense that the emergence of this hybrid imaging modality was driven by technology and the need to address many interesting and challenging scientific questions to make the combination of the two imaging modalities possible from the instrumentation perspective, whereas the clinical applications remained unexplored and not yet established. Indeed, during the last decade, a large number of publications reported on innovative developments in MR-compatible PET detectors and various strategies to reduce the interference between the two imaging modalities. Physicists had also to rethink the issue of attenuation correction and address the many challenges of MR imaging-guided attenuation correction, particularly in whole-body PET/MR imaging. As such, this technology was regarded as "a solution looking for a problem" 5 rather than a solution to an existing problem. Indeed, despite much worthwhile research and development efforts focusing on solving technical issues, the success of any medical imaging modality is not measured by the degree of technical complexity and innovation aspect that led to its maturity. ${ }^{6}$ It is rather measured by the sensible advantages and potential clinical benefits that this technology is going to bring in the clinical setting, keeping in mind that these have to be weighed against the substantial increase in costs and complexity for its implementation in clinical routine.

This issue of PET Clinics addresses the subject of hybrid PET/MR imaging technology, covering advances in both instrumentation and quantitative imaging procedures developed to address the 
above described challenges. Following the initial introduction and current commercial availability of this technology from the main leading players, offering different platforms for sequential and simultaneous PET/MR imaging, the role and limitations of this modality in clinical setting are being debated, particularly for applications in cardiology and oncology. Overall, there is a wide consensus that while this technology is still in its infancy and did not reach the required level of maturity for widespread clinical adoption, it is considered a promising advance that seems to have the potential to overcome some of the deficiencies of existing hybrid imaging modalities. During the next decade, additional exciting technical developments are foreseen and large-scale multicenter clinical trials are expected to answer critical questions regarding the clinical benefits of this technology.

In this issue of PET Clinics, a number of pertinent areas anticipated to play a pivotal role and have a substantial impact on the future of PET/ MR imaging are highlighted. This includes advanced topics in preclinical and clinical PET/ $M R$ instrumentation and dual-modality contrast agents, MR imaging-guided partial volume and attenuation correction, including strategies to compensate for attenuation from rigid and nonrigid MR coils, as well as advanced MR imaging protocols for improved derivation of the arterial input function for PET kinetic modeling applications. It is hoped that this collection of comprehensive topics in hybrid PET/MR imaging will be useful to readers interested in both instrumentation and quantitative imaging protocols as well as their applications in clinical and research settings.

Habib Zaidi, PhD Division of Nuclear Medicine and Molecular Imaging Geneva University Hospital $\mathrm{CH}-1211$ Geneva, Switzerland

E-mail address: habib.zaidi@hcuge.ch

\section{REFERENCES}

1. Mayneord WV. The radiology of the human body with radioactive isotopes. Br J Radiol 1952;25:517-25.

2. Kuhl DE, Hale J, Eaton WL. Transmission scanning: a useful adjunct to conventional emission scanning for accurately keying isotope deposition to radiographic anatomy. Radiology 1966;87:278-84.

3. Anger HO, McRae J. Transmission scintiphotography. J Nucl Med 1968;9:267-9.

4. Torigian DA, Zaidi H, Kwee TC, et al. PET/MR imaging: technical aspects and potential clinical applications. Radiology 2013;267:26-44.

5. Yankeelov TE, Peterson TE, Abramson RG, et al. Simultaneous PET-MRI in oncology: a solution looking for a problem? Magn Reson Imaging 2012;30:1342-56.

6. Wehrl HF, Sauter AW, Divine MR, et al. Combined PET/MR: a technology becomes mature. J Nucl Med 2015;56:165-8. 